\title{
Programa de Aprendizaje en Multigrado: una experiencia de mejora educativa en el estado de Puebla
}

\section{Multigrade Learning Program: an experience about how to improve education in the State of Puebla}

Fernando Mejía, Erika Argándar, Margarita Arruti, Adriana Olvera, María del Mar Estrada*

RESUMEN

A lo largo y ancho del planeta hay suficientes experiencias exitosas de educación multigrado que demuestran que esta no puede ser atendida desde la perspectiva de escuelas incompletas (Little A., 2006). En sí mismo el multigrado es un modelo educativo, que entre otras, ha enseñado a las escuelas graduadas elementos importantes (trabajo colaborativo, adecuación de aulas para acceso a materiales, entre otras).

El Programa de Aprendizaje en Multigrado (PAM) es un modelo de aprendizaje que busca la mejora educativa en escuelas de organización multigrado, que ocupan un lugar importante dentro del Sistema Educativo Nacional y que constituyen una proporción considerable de escuelas en el país. Se gesta en el estado de Puebla, a partir de la implementación del modelo colombiano Escuela Nueva, y se adapta retomando las experiencias exitosas que las y los docentes mexicanos realizan en su trabajo cotidiano. El PAM ha transitado por distintas etapas, y los resultados de las evaluaciones que se le han realizado evidencian un impacto favorable en la organización y gestión escolar, así como en el aprendizaje del alumnado.

Palabras clave: escuelas multigrado, aprendizaje en multigrado, modelo educativo, mejora educativa

\section{ABSTRACT}

Throughout the planet, there are successful experiences of multigrade education that demonstrates how that kind of schools should not be attended as incomplete (Little, 2006). Multigrade schools have an specific educational model, which can taught to graduated schools important elements like work collaborative, adequacy of classrooms for access to materials, among others.

PAM is a learning model that seeks educational improvement in multigrade schools organization, which get an important place within the national education system and constitute a significant proportion of schools in the country. It appeared in the State of Puebla, starting from the implementation of the Colombian model Escuela Nueva. It was adapted recovering successful teaching experiences coming from Mexican teachers. PAM has gone through into different stages, and the results of the evaluations have demonstrated a positive impact on the organization and school management, as well as the learning of students.

Key words: multigrade schools, learning in multigrade, educational model, educational improvement

\footnotetext{
*Centro de Estudios Educativos: fernandomejia@cee.edu.mx; adriolvera@yahoo.com.mx; maria.del.mar.estrada@gmail.com. Fundación Azteca: eargandarc@tvazteca.com.mx; margarita699gmail.com
} 


\section{INTRODUCCIÓN}

El Programa de Aprendizaje en Multigrado (PAM) es un modelo educativo para escuelas de organización multigrado que surge de la conjunción de formas de trabajo y propuestas pedagógicas exitosas. Este programa fue diseñado por la Secretaría de Educación del estado de Puebla, con el apoyo de Fundación Azteca, A. C., a fin de contribuir a elevar la calidad de la educación en las escuelas.

Los resultados de la implementación del programa, hasta este momento, muestran que contribuye de manera significativa a mejorar la calidad de la educación en las escuelas, pues propicia profundas transformaciones en la institución escolar.

En el presente trabajo se presentan los antecedentes del programa, las etapas por las que ha transitado, características, beneficiarios, recursos didácticos y de aprendizaje, así como los resultados que ha obtenido; se plantean, además, algunas sugerencias de expansión del modelo.

\section{ANTECEDENTES}

Las escuelas de organización multigrado son aquellas donde un docente atiende a más de un nivel en un mismo grupo. De acuerdo con Estrada (2015), existen dos tipos de escuelas multigrado (para los niveles preescolar, primaria y secundaria): las estatales y federales generales atendidas por docentes, y los cursos comunitarios desplegados en comunidades pequeñas, los cuales dependen del Consejo Nacional para el Fomento Educativo (CONAFE), que son atendidos por líderes educativos comunitarios, bajo un modelo y con materiales distintos de los de las escuelas generales.

El trabajo en las escuelas generales presenta retos importantes, ya que estas no cuentan con un modelo propio, y ello provoca que hagan adaptaciones a lo dispuesto para las escuelas de organización completa (ibid.). Los docentes, por ejemplo, cumplen de manera simultánea con tareas frente a grupo y de dirección escolar, atienden alumnado de diferentes grados de manera sincrónica; estas escuelas no cuentan con personal de apoyo y, con ello, se hacen cargo de tareas administrativas y de mantenimiento, todo 
esto en el mismo tiempo escolar, lo que les disminuye disponibilidad para su quehacer pedagógico.

A pesar que las escuelas multigrado constituyen una pieza fundamental en el país, no existe una categoría como tal dentro del Sistema Educativo. De acuerdo con el INEE (2015), para el ciclo escolar 2013-2014, había 26.8\% de escuelas de preescolar unitarias, mientras que para primaria $44 \%$ formaban parte de las escuelas de organización multigrado. ${ }^{1}$ Cabe destacar que 11 entidades cuentan con más de $50 \%$ de escuelas primarias multigrado. Los estados con los porcentajes más altos son San Luis Potosí (60.8\%), Durango (62.8\%), y Chiapas (69.9\%). ${ }^{2}$ Aproximadamente, uno de cada 10 niños y niñas estudia en primarias multigrado.

En el caso del nivel secundario, las telesecundarias son las que pueden presentar una organización multigrado, y así lo es para $19.7 \%$, de las cuales $7.9 \%$ son unitarias y $11.8 \%$ son bidocentes.

En particular, en las escuelas indígenas, los porcentajes aumentan considerablemente así que, en preescolar, 49.9\% son unitarias (un solo docente atiende a los tres grados). En primaria $66.5 \%$ son multigrado (INEE, 2016: 120). Se las considera escuelas "incompletas" porque no tienen nómina docente para cada grado, y alguien del cuerpo docente cumple con las funciones de dirección.

No obstante, sus características representan ventajas educativas, pues esta diversidad de niveles y de edades dentro del aula favorece el aprendizaje colaborativo entre el alumnado, un mayor involucramiento de los padres de familia y una mayor contextualización. Las condiciones de simultaneidad de funciones hacen posible que la gestión escolar y áulica sean más "porosas" entre ellas, lo que facilita que la gestión pedagógica gire alrededor de lo pedagógico de manera más fluida.

A lo largo del tiempo ha habido algunos intentos de reorganización del currículo oficial para adaptarlos a la escuela multigrado, siendo la Propuesta Educativa Multigrado 2005 (PEM05)

\footnotetext{
${ }^{1}$ Tanto para preescolar como para primaria se consideran los servicios general, indígena y comunitario.

${ }^{2}$ En particular, en el estado de Puebla, $43.1 \%$ de escuelas primarias son de organización multigrado.
} 
la más importante y, aunque es un documento muy valioso que aporta orientaciones, formas de trabajo en el aula y una organización del currículo que permite abordar un mismo tema en diferentes niveles simultáneamente y que, aún hoy en día, muchos docentes utilizan como referencia, no plantea acciones de capacitación y de acompañamiento a los docentes para que puedan operativizar esta propuesta en las aulas, además de no atender la dimensión escolar como tal.

Interesado por ensayar una propuesta específica para este sector, tradicionalmente desatendido, el gobierno del estado de Puebla solicitó el apoyo de Fundación Azteca para probar un modelo de aprendizaje que contribuyera tanto a atender sus particularidades como a potenciar sus ventajas educativas.

Derivado de este interés, se inicia en la región de Teziutlán, Puebla, en octubre de 2012, una prueba piloto en 20 escuelas primarias multigrado con el modelo Escuela Nueva de Colombia.

Este modelo fue diseńado a mediados de los setenta para ofrecer la primaria completa y mejorar la calidad y efectividad de las escuelas rurales, especialmente las multigrado, por ser las más necesitadas y aisladas del país. Dichas escuelas comparten características similares con las mexicanas. Mediante estrategias e instrumentos concretos, este modelo promueve un aprendizaje activo, participativo y colaborativo, así como el fortalecimiento de la relación escuela-comunidad, logrando modificar el modelo educativo convencional centrado en el docente, hacia uno enfocado en el estudiante; donde la escuela/aula es la unidad fundamental de cambio para mejorar la cobertura, la calidad y la equidad de la educación.

Es por todas estas características que Fundación Azteca acerca este modelo al estado, lo que permitió, al concluir la fase piloto y considerando los resultados obtenidos, llegar a una adaptación local del modelo colombiano, incorporando otras estrategias exitosas mexicanas, lo que dio lugar al ahora denominado Programa de Aprendizaje en Multigrado (PAM), el cual se ha expandido a otras modalidades y niveles en la región de Teziutlán (primarias generales e indígenas, así como telesecundarias), afianzándose en la zona gracias al compromiso de docentes y de miembros de la mesoestructura. 


\section{COMPONENTES PEDAGÓGICOS DEL PROGRAMA}

Si bien el modelo tiene como base Escuela Nueva, se adaptó y complementó con estrategias de modelos mexicanos que los docentes habitualmente despliegan en su quehacer pedagógico. Los complementos son de metodologías propias de cada nivel y modalidad, donde las estrategias y los instrumentos que los docentes ya trabajan cobran una nueva dimensión, articulándose con el currículo y dinamizándolo. Cabe destacar que el modelo requiere de un trabajo colegiado para su implementación y de una adaptación a las circunstancias de cada escuela para que funcione de manera óptima.

Su propósito es llevar la dinámica del aula y la escuela hacia la participación del alumnado en su proceso de aprendizaje, fomentando un esquema de trabajo que enfatice la vivencia cotidiana de procedimientos y mecanismos participativos, a través de ambientes de aprendizaje adecuados, responsabilidad compartida y prácticas democráticas. Asimismo, promueve un cambio de rol docente en la gestión del aprendizaje en el aula y la escuela, fortaleciendo y motivando formas positivas de relación con la comunidad para garantizar su apoyo a las escuelas, a fin de que el aprendizaje se dé en óptimas condiciones y se mejore la gestión escolar.

También promueve el desarrollo de habilidades clave como la toma de decisiones, la planeación y organización de actividades, la comunicación efectiva, el trabajo en equipo, el pensamiento crítico, aprender a aprender, entre otras. Sus principales características son las siguientes: 


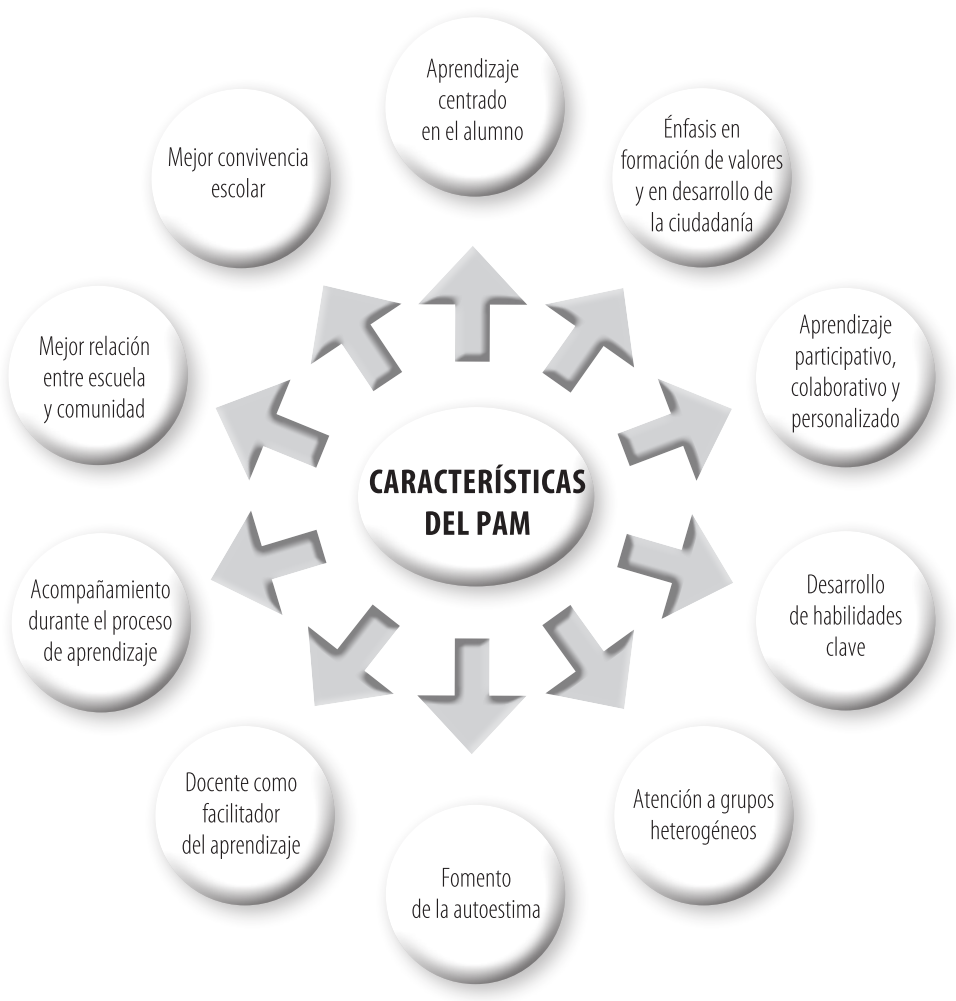

Por otro lado, el PAM apunta a la articulación de la formación académica del alumnado con su desarrollo afectivo, social y moral, con la finalidad de que tenga una formación integral.

También intenta fortalecer el desempeño escolar utilizando una pedagogía activa, mediante cuatro elementos que dan identidad al Programa: Participación democrática, Ambiente propicio para aprender, Relación escuela-comunidad y Colectivo docente.

A través de la Participación democrática se pretende desarrollar y reconocer las diferentes capacidades de los integrantes de un grupo, para lo cual se considera una serie de procedimientos y mecanismos democráticos que enfatizan la participación, la toma de decisiones colectiva y compartida, la libertad de elección y opinión, diferentes niveles de responsabilidad, aporte de ideas y trabajo conjunto. 
El propósito del elemento denominado Ambiente propicio para aprender es crear ambientes con las condiciones básicas para aprender con gusto y para desarrollar capacidades, habilidades y actitudes, donde prevalezcan relaciones armónicas entre docente y estudiantes. En este elemento es fundamental la mejora en la relación entre alumnado

Respecto a la Relación escuela-comunidad, su finalidad es fortalecer la relación entre la escuela y la comunidad en favor de las y los niños y de su aprendizaje, mediante estrategias que permiten acercarse a ella, conocerla y organizarla.

Finalmente, el propósito del elemento Colectivo docente es fortalecer el quehacer formador de los docentes y enriquecerlo, reuniéndolos para hablar de lo pedagógico, intercambiar saberes, dudas y experiencias sobre el modelo, lo que también implica un trabajo colegiado al interior de la escuela.

Estos elementos se ponen en funcionamiento a través de diversos instrumentos. En el siguiente esquema se exponen los instrumentos correspondientes a cada elemento.

\section{COMPONENTES PEDAGÓGICOS DEL PAM}

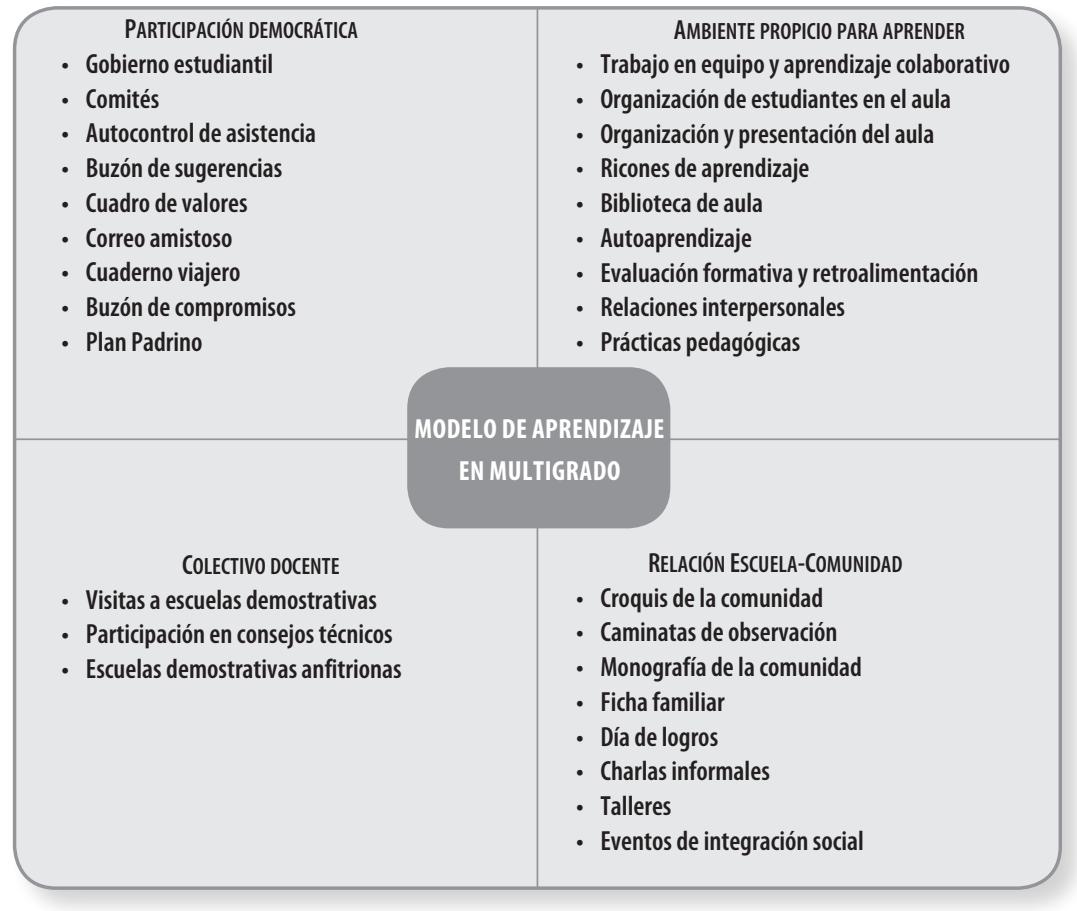




\section{Materiales diseñados}

Para el desarrollo del PAM en las escuelas se diseñaron materiales conforme se trabajaba en la implantación. A continuación se describen:

1. Guia de gestión escolar y de aula: contiene los fundamentos del modelo multigrado que propone y el Programa en el que se enmarca la propuesta; el conjunto de orientaciones para docentes que permiten desarrollar el modelo en el aula y las ideas para la planeación, el seguimiento y la evaluación. Es fundamental durante la impartición del taller de inducción, y también es la herramienta para la introducción, el uso y la apropiación del modelo en las escuelas.

2. Instrumento para el fortalecimiento de la escuela multigrado: cuyo propósito es recuperar información de la ejecución del modelo a nivel escolar para determinar su estatus y poder proporcionar una retroalimentación pertinente y oportuna, con fines de apropiación del modelo por parte de la totalidad de la comunidad escolar.

3. Bitácora PAM: es un software que ayuda a documentar y valorar el acontecer del PAM en la escuela y que proporciona informes de avances en cada uno de los componentes del programa, además de apoyar el proceso de seguimiento y acompańamiento. Se propone que el instrumento y la Bitácora se utilicen en dos partes: la primera, de acompańamiento, que implica la visita a las escuelas, y la segunda, de seguimiento, que se desarrolla propiamente en los Consejos Técnicos.

Estos materiales favorecen el trabajo de implantación del PAM en las escuelas, brindan apoyo directo a los planteles (la Guía) y facilitan el seguimiento y acompańamiento (supervisores y ATP). Es importante anotar que la Guía fue elaborada conjuntamente con docentes, ATP, supervisores y jefes de sector que ya desarrollaban el Programa; lo anterior permitió un material de fácil uso, y muy bien valorado por el cuerpo de docentes. El instrumento de fortalecimiento y la bitácora también han sido útiles, pues los validadores fueron los mismos supervisores y ATP. 
Desde el inicio se tuvo la intención de que el PAM fuera bien acogido por los actores del sistema educativo en Puebla; por eso se decidió que fueran ellos mismos quienes impartieran la formación. Para ello se diseñó un taller de formación de formadores, y se desarrollaron materiales para fortalecer el taller de inducción. Estos contienen las voces de los agentes involucrados ya que han tenido una participación relevante en su creación.

1. Taller de formación de formadores: agenda y carta descriptiva que contienen la secuencia didáctica de la capacitación: propósitos, contenidos, tiempos y actividades a realizar. Incluye el correspondiente material didáctico de apoyo.

2. Taller de inducción: agenda, carta descriptiva de capacitación y presentación de apoyo a la impartición del taller de inducción. Asimismo, se han desarrollado unas fichas pedagógicas sobre cada una de las estrategias e instrumentos del modelo, para que los facilitadores tengan información resumida y con ejemplos durante la impartición del taller.

3. Taller de seguimiento y acompañamiento: agenda y carta descriptiva de capacitación y presentación de apoyo, así como algunos documentos con orientaciones para la integración del PAM en las Rutas de mejora.

4. Manual para escuelas demostrativas: documento que contiene los criterios para seleccionar a las escuelas demostrativas y las normas a seguir para visitarlas. Asimismo, plantea el protocolo de atención por parte de estas escuelas y orientaciones precisas para la observación por parte de los visitantes. ${ }^{3}$

\section{Referentes operativos del PAM}

La implementación del PAM en las escuelas pasa por tres etapas, a través de las cuales se observa el grado de incorporación de cada uno de los instrumentos en el aula y la vida escolar.

\footnotetext{
${ }^{3}$ Cabe señalar que esta estrategia, que forma parte del componente Colectivo Docente, no ha podido instalarse a la fecha, ya que aún se están identificando los mecanismos para no interferir con la normalidad mínima.
} 
1. De la formación inicial en el modelo, a la introducción de los instrumentos y estrategias en el aula.

2. De la introducción, al uso continuo de dichos instrumentos/ estrategias y su fusión con el trabajo en el aula.

3. Del uso continuo, a la apropiación del modelo, cuando la escuela lo adapta para la atención de sus necesidades y donde hay un cambio de actitud y de comportamiento tanto en los docentes como en el estudiantado, así como una mayor participación de la comunidad.

Para implementar el PAM ha sido necesaria la puesta en marcha de las fases del modelo y el desarrollo de materiales de trabajo. Las fases del modelo resultan de la estrategia piloto en las 20 escuelas y de las subsecuentes expansiones a otras escuelas de la región de Teziutlán, y son las siguientes:

\section{Fases del modelo}

1. Selección y formación de un grupo de docentes, asesores técnico pedagógicos, supervisores y jefes de sector como facilitadores del taller de inducción a un nuevo cuerpo docente.

2. Impartición del taller de inducción al nuevo cuerpo docente y demás miembros de la mesoestructura involucrada. Este tiene una duración de 40 horas, y sigue una metodología vivencial para preparar al cuerpo docente en la introducción gradual del modelo en sus escuelas.

3. Capacitación de la mesoestructura (jefes de sector, supervisores y asesores técnico pedagógicos) en las funciones de seguimiento y acompañamiento del PAM en las escuelas.

4. Incorporación del Programa en las Rutas de mejora de las escuelas, a fin de articular sus acciones y estrategias con las que estén planteadas para que se aborden como algo integral.

5. Integración del PAM en los Consejos Técnicos Escolares, a fin de aprovechar este espacio también para la formación de nuevos maestros (sin que esto sea en sustitución del taller de inducción), para intercambiar experiencias y resultados, así como para dar seguimiento a la implementación del programa. 
6. Implementación de las estrategias e instrumentos del modelo al trabajo en las aulas y en las escuelas.

7. Visitas de seguimiento y acompańamiento a los docentes en sus escuelas, a fin de retroalimentarles y orientarles para el mejor desarrollo del PAM.

\section{Etapas de implementación}

El PAM ha transitado, desde 2012 hasta la fecha, por cuatro etapas. En cada una de estas el aprendizaje constante y los resultados de las diversas evaluaciones han posibilitado su instalación $-\mathrm{y}$ por lo tanto, su expansión- en escuelas de diversas modalidades y niveles educativos en el estado de Puebla. Para que esto fuese posible, han sido pieza fundamental la SEP Puebla y Fundación Azteca, así como la participación (en la primera etapa) de la Fundación Escuela Nueva de Colombia y la colaboración del Centro de Estudios Educativos (CEE), institución encargada de monitorear y evaluar el Programa a lo largo de este tiempo.

\section{Prueba piloto (ciclos escolares 2012-2013 y 2013-2014)}

En el ciclo escolar 2012-2013 se inició una prueba piloto que consistió en la implementación del modelo Escuela Nueva de Colombia en 20 escuelas primarias generales de la zona de Teziutlán, Puebla, la cual concluyó en el ciclo escolar 2013-2014.

Durante este periodo, se llevaron a cabo las siguientes acciones:

- Pasantía en Colombia (octubre de 2012), en la que participaron docentes, asesores técnico pedagógicos y supervisores de la zona señalada, cuya finalidad fue que los asistentes pudieran conocer de cerca el modelo y las escuelas rurales que lo trabajan.

- Posteriormente, la Fundación Escuela Nueva impartió dos talleres a los docentes de esas escuelas: uno de inducción y otro relacionado con el manejo y uso de guías de aprendizaje. ${ }^{4}$

${ }^{4}$ El modelo, en Colombia, trabaja con unas guías de aprendizaje basadas en los estándares básicos de competencia colombianos, las cuales son una parte medular del modelo y apoyan la planeación de clases y la evaluación de los aprendizajes. 
- Se dio una asesoría por parte de esta Fundación para el montaje y ambientación de las aulas y se llevaron a cabo visitas de seguimiento, monitoreo y retroalimentación a las escuelas participantes.

- Se establecieron tres Microcentros, considerando la cercanía geográfica de las escuelas, y se designó a un supervisor para la coordinación de cada uno de ellos. Los Microcentros son una estrategia importante del modelo Escuela Nueva y constituyeron un espacio para la formación, retroalimentación y el intercambio de experiencias entre los docentes y la mesoestructura.

- El Centro de Estudios Educativos (CEE) llevó a cabo el monitoreo y una evaluación Pre y Postest en estas escuelas.

- Las 20 escuelas y sus equipos de supervisión continuaron con la dinámica de trabajo durante el ciclo escolar 2013-2014.

\section{Primera etapa de expansión (ciclo escolar 2014-2015)}

En 2014, se decidió diseñar un modelo propio para Puebla, con lo cual surge el Programa de Aprendizaje en Multigrado, que integró los resultados obtenidos en la prueba piloto con Escuela Nueva, la experiencia de la escuela multigrado en México y la normatividad de la Secretaría de Educación Pública.

Para esta primera etapa de expansión, se integraron 97 escuelas, todas de la región de Teziutlán, incorporando ahora a primarias indígenas y telesecundarias. Con ello se benefició a 4264 estudiantes. Aquí, se buscó consolidar la estrategia de formación mediante un taller de formación de formadores, durante el cual se diseñó el programa para el taller de inducción.

Como parte de las estrategias y acciones emprendidas durante el diseño del PAM, se elaboraron y desarrollaron los materiales e instrumentos ya presentados en el apartado anterior. Asimismo, se aprovecharon los cambios que la SEP dispuso para los Consejos Técnicos, para llevar a ellos las dinámicas y aprendizajes derivados de la estrategia de Microcentros, aplicada durante la prueba piloto con Escuela Nueva.

El Centro de Estudios Educativos realizó el levantamiento de información Pretest y Postest para identificar y evaluar los 
cambios que presentaron las escuelas participantes. Esta evaluación incluyó el tema de actitudes docentes.

En este mismo periodo, se llevó a cabo el primer taller de seguimiento y acompañamiento dirigido a supervisores y jefes de sector, el cual se apoya en el documento de orientaciones para incorporar el PAM en las rutas de mejora, en el instrumento para el fortalecimiento de las escuelas multigrado y en la Bitácora PAM. Durante este taller se realizó el primer ejercicio de integración del Programa en las rutas de mejora.

Así mismo, en el periodo de instalación del PAM en las 97 escuelas, se llevaron a cabo visitas de seguimiento y retroalimentación a los establecimientos escolares.

\section{Segunda y tercera etapas de expansión PAM (ciclo escolar 2015-2016)}

La segunda etapa de expansión, realizada en el ciclo escolar 2015-2016, consideró, por un lado, en el primer tercio de 2015, un taller de formación de formadores para ampliar el equipo de facilitadores del taller de inducción, y por otro, un taller de inducción a docentes de 57 escuelas de tiempo completo en dos sedes: Tlatlauquitepec y Zacapoaxtla, durante el receso académico de agosto de 2015.

Como en los ciclos anteriores, se efectuaron visitas aleatorias de seguimiento y acompañamiento a las escuelas y, en esta ocasión, también se hicieron visitas de observación a algunos Consejos Técnicos, para identificar las estrategias que se están siguiendo respecto al PAM y la manera en que están integrando este a sus rutas de mejora.

Durante la tercera etapa de expansión, llevada a cabo en este mismo ciclo escolar, se desarrollaron las siguientes acciones:

- Impartición de una taller de inducción a 56 escuelas de organización completa, llevado a cabo en febrero de 2016 en la ciudad de Teziutlán, con un total de 210 escuelas atendidas, a fin de identificar las posibles adecuaciones que deben hacerse al modelo para este tipo de escuelas, ya que sus condiciones y características son diferentes de las de multigrado. 
- Dos reuniones de trabajo con el equipo de formadores, para identificar acciones encaminadas a la mejora continua del taller de inducción al PAM. El resultado fue el desarrollo de las fichas pedagógicas y de una presentación estándar para apoyar la impartición del taller de inducción. Asimismo, se identificaron y acordaron estrategias, tanto para incentivar el uso de la Bitácora, como para integrar el PAM en los Consejos Técnicos Escolares.

- Visitas de seguimiento a escuelas participantes en diversas etapas de implementación del PAM, así como a las nuevas escuelas capacitadas hacia el final del ciclo escolar, a fin de darles tiempo de iniciar la implantación.

- Visitas de observación de la integración del PAM en Consejos Técnicos Escolares en escuelas capacitadas en etapas previas, y de algunas participantes en esta última etapa de expansión.

\section{RESULTADOS DE LA IMPLEMENTACIÓN}

A la fecha, 210 escuelas han integrado el PAM en su labor, beneficiando a 24993 alumnos y a 773 docentes, de acuerdo con la siguiente distribución:

\begin{tabular}{|c|c|c|c|c|}
\hline No. escuelas & Nivel & Modalidad & No. docentes & No. estudiantes \\
\hline 106 & Primarias & Generales & 496 & 16265 \\
\hline 58 & Primarias & Indígenas & 120 & 4422 \\
\hline 46 & Secundaria & Telesecundaria & 157 & 4306 \\
\hline 210 & & & 773 & 24993 \\
\hline
\end{tabular}

De acuerdo con el monitoreo y la evaluación Pretest (2014) y Postest (2015) del Programa, a cargo del CEE, el modelo suscita vivencias y aprendizajes nodales en sí mismos, que son condición de posibilidad para un mejor aprendizaje en general. No son ajenos o adicionales al modelo educativo ni al currículo vigente: están alineados con ellos, y ayudan a concretar asuntos que, a pesar de estar contemplados en el currículo, no suelen hacerse operativos de forma sistemática.

En el levantamiento de información correspondiente al Pretest participaron 50 escuelas, mientras que en el Postest fueron 103. 
En la siguiente tabla se muestra la distribución de escuelas participantes por momento de evaluación, etapa de implementación, y modalidades educativas.

\section{Escuelas participantes en los levantamientos Pretest-Postest}

\begin{tabular}{|c|c|c|c|}
\hline & \multirow{3}{*}{$\begin{array}{c}\text { Pretest (2014) } \\
\text { Escuelas }\end{array}$} & \multirow[b]{2}{*}{ Postest (2015) } \\
\hline & & & \\
\hline & & & Escuelas \\
\hline Piloto & Primarias generales & 18 & 20 \\
\hline \multirow{2}{*}{ Ampliación pretest } & Primarias generales & 16 & 16 \\
\hline & Primarias indígenas & 16 & 16 \\
\hline \multirow{2}{*}{ Ampliación postest } & Primarias generales & \multirow{2}{*}{ No participaron } & 25 \\
\hline & Telesecundarias & & 19 \\
\hline \multirow{2}{*}{ Adicionales } & Primarias generales & \multirow{2}{*}{ No participaron } & 2 \\
\hline & Telesecundarias & & 5 \\
\hline & Totales & 50 & 103 \\
\hline
\end{tabular}

Los instrumentos de recolección de información fueron los siguientes:

- Cuestionario para el cuerpo docente.

- Cuestionario para el alumnado.

- Guía de observación y registro.

- Test de Autoestima Escolar.

- Test de Comportamiento Democrático.

- Análisis de la Bitácora.

- Cuestionario de Actitudes Docentes.

A continuación se presentan los resultados derivados del examen de cada uno de los componentes del modelo. En el componente de Participación democrática, se encontró que los Gobiernos Estudiantiles y los Comités están en marcha prácticamente en todas las escuelas. Hay una alta participación estudiantil, aunque queda pendiente -como área de mejora- fortalecer las acciones de acompańamiento por parte de docentes. Los instrumentos y estrategias de este elemento se encuentran presentes en casi todas las aulas, aunque con diferencias en la instalación y apropiación, ante lo cual resulta necesario el refuerzo de la intencionalidad pedagógica. 
Porcentajes de uso de los instrumentos/herramientas, 2014-2015

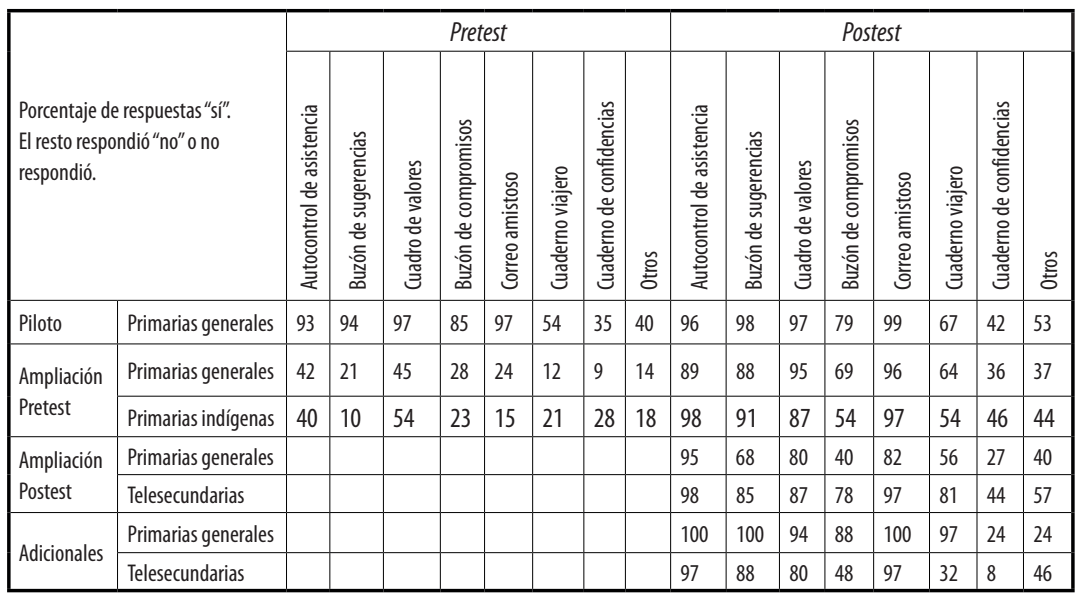

En el elemento Ambientes propicios para aprender, el PAM ha logrado promover una mayor versatilidad en la organización de las y los estudiantes en el aula, en detrimento de la rigidez y unidireccionalidad del acomodo permanente por filas, para transitar a uno más frecuente por grupos. El PAM también ha propiciado más experiecias de trabajo por grupos, con un marcado aumento en los diferentes roles (monitoreo, gestión de materiales, control del tiempo, relatoría y mediación). Los Rincones de aprendizaje, que cuentan con preexistencias importantes, con el PAM llegan a más de $80 \%$ de presencia en las aulas. Las nińas y niños de escuelas indígenas consultan estos y otros materiales con mayor frecuencia. Las aulas están ambientadas con los diferentes instrumentos y demás materiales, aunque sigue pendiente que estos sean más pertinentes, para lo que se está trabajando, y que estén organizados a fin de no provocar saturación.

El elemento Relación escuela-comunidad presenta el principal reto. Sus instrumentos y estrategias han sido introducidos y apropiados en menor medida (el Croquis de la comunidad es la excepción). El Test de Comportamiento Democrático no revela avances en este rubro. Las y los docentes reportan que la participación de familiares en las actividades escolares se da con regularidad, y hay visitas ocasionales de los primeros a los segundos; 
pero ambos temas permanecen sin avances. El profesorado tiene actitudes más bien favorables hacia el trabajo con familiares y hacia la integración escuela-comunidad, aunque con ciertos límites.

Respecto al Colectivo docente, se encontró que las y los docentes valoran bien el Consejo Técnico como espacio de aprendizaje e intercambio en el PAM, y se reporta que este constituye un espacio importante de formación. En algunos casos se activa el acompańamiento en el programa por parte de los mismos docentes. A la mayoría le interesaría visitar Escuelas demostrativas, y a la mitad, que sus escuelas sean anfitrionas, a pesar de que la estrategia no está oficialmente en marcha y desconocen los detalles de su funcionamiento.

Las evaluaciones también han arrojado resultados sobre los referentes operativos del PAM. Al respecto se destaca lo siguiente.

Alrededor de la mitad de maestras y maestros considera que la formación recibida en el PAM les brinda herramientas suficientes para desarrollarlo; el resto estima que solo en parte, y la minoría considera que no. Muchos expresan la necesidad de una formación continua para conocer aplicaciones concretas del programa, disipar dudas, intercambiar experiencias, etc. Asimismo, ven necesario que se forme a quienes no asistieron al Taller de inducción (que llega a ser la mitad de los maestros de las primarias generales de la ampliación postest).

La Guía para la gestión escolar y de aula juega un papel importante dentro la escuela y el PAM, y la mayoría de las y los docentes considera que su contenido es claro y útil para implementarlo. Aunque en muchas escuelas no se reportan problemas por motivo de los insumos necesarios para el programa, muchos docentes expresan las dificultades propias y de las familias para aportarlos, dadas las limitaciones materiales de las comunidades.

Alrededor de la mitad de las escuelas y docentes recibieron acompańamiento y seguimiento con el apoyo de la Bitácora. Sin embargo, disminuyó el porcentaje de docentes que reportan ser acompañados en lo general (en el contexto de la drástica disminución de ATP durante el ciclo 2015-2016). El acompañamiento en el PAM corre a cargo de la supervisión, y en menor medida, del ATP u otros docentes. El análisis de los reportes generados desde la Bitácora, que se realizaron en dos etapas durante 
el ciclo escolar 2014-2015, muestra que el promedio de avance de los instrumentos de trabajo cotidiano son los más altos, por ejemplo, Autocontrol de asistencia (Participación democrática) y Biblioteca del aula (Ambientes propicios para aprender). Asimismo, las escuelas participantes en el Piloto mostraron los promedios más altos en el avance de implementación, lo que evidencia que el trabajo constante con las herramientas favorece la instalación del modelo en las escuelas.

La mayoría de maestros y maestras tiene en alta valía al PAM; en conjunto, estiman que este contribuye a su superación profesional como docentes (70\%), y que beneficia el aprendizaje de las y los niños (90\%). Tienden a considerar que el programa es parte de su identidad profesional (alrededor de 70\%), y aún más, que es parte de la identidad de sus colectivos docentes (más de $80 \%)$. A pesar de los retos que implica su nuevo rol, sus actitudes frente al PAM tienden a ser favorables. Más que un programa, es un proyecto que cobra sentido y fuerza en la medida del compromiso, la convicción y la colaboración entre los distintos actores involucrados: docentes, estudiantes, familiares, ATP, supervisores, jefes de sector, funcionarios, autoridades educativas locales y otras instancias promotoras.

El hecho de involucrar a la mesoestructura desde la formación ha sido un acierto; sus integrantes son un factor crítico de éxito en la implementación del programa. El PAM ha desarrollado un modelo operativo donde la formación de los docentes y el seguimiento al programa está a cargo de los mismos actores del sistema (supervisores y ATP). En el seguimiento y acompañamiento, los docentes, en sus Consejos Técnicos, determinan cómo implementarán el PAM en sus escuelas, y cómo este apoyará los objetivos establecidos en sus Rutas de Mejora. Los supervisores y ATP dan acompańamiento y seguimiento al cuerpo docente mediante visitas y a través de su liderazgo en los Consejos Técnicos. Ahí, el Colectivo Docente intercambia sus experiencias, retroalimenta sus prácticas y ajusta sus objetivos.

El PAM está en marcha en el conjunto de las escuelas piloto (pioneras y forjadoras del programa desde el ciclo 2012-2013), así como en las que formaron parte de las siguientes ampliaciones y en algunas adicionales a la lista oficial. A continuación se 
presenta un cuadro donde se describe cómo estaban las escuelas en su implementación, al final de su primer ciclo escolar de ampliación (2014-2015). La implementación y apropiación se ha dado en magnitudes variables:

Implementación y apropiación del PAM en las escuelas, diferentes etapas

\begin{tabular}{|c|c|c|}
\hline Etapa & Tipo y número de escuelas & Descripción de la implementación \\
\hline Piloto & Primarias generales (20) & $\begin{array}{l}\text { En general, se mantiene en marcha el PAM, aunque hay poca } \\
\text { evidencia de avances en la apropiación. }\end{array}$ \\
\hline \multirow{2}{*}{ Ampliación pretest } & Primarias generales (16) & $\begin{array}{l}\text { Aunque con áreas de oportunidad, han llevado a cabo la } \\
\text { implementación del PAM en buena medida. }\end{array}$ \\
\hline & Primarias indígenas (16) & $\begin{array}{l}\text { Han llevado a cabo la implementación del PAM en gran medida, y } \\
\text { el impacto en la vida escolar ha sido drástico. }\end{array}$ \\
\hline \multirow[t]{2}{*}{ Ampliación postest } & Primarias generales (25) & $\begin{array}{l}\text { Implementación comparable en primarias generales de la } \\
\text { ampliación pretest, aunque varios aspectos del uso y apropiación } \\
\text { están ligera o significativamente por debajo. }\end{array}$ \\
\hline & Telesecundarias (19) & $\begin{array}{l}\text { Excelentes niveles en la implementación; muchos, mejores que en } \\
\text { las primarias (excepto en Relación Escuela-Comunidad). }\end{array}$ \\
\hline \multirow{2}{*}{ Adicionales } & Primarias generales (2) & $\begin{array}{l}\text { Cuentan con muchos de los instrumentos y estrategias, pero varios } \\
\text { no están en marcha o con magros efectos, mientras que algunos } \\
\text { muestran mejores resultados que el resto. }\end{array}$ \\
\hline & Telesecundarias (5) & $\begin{array}{l}\text { Cuentan con los instrumentos en sus aulas, y su desempeño es } \\
\text { bueno: en casi todos los aspectos están ligeramente a la zaga de las } \\
\text { telesecundarias ampliación postest. }\end{array}$ \\
\hline Adicionales bitácora & $\begin{array}{l}\text { Primaria general ( } 1) \text {, } \\
\text { indígenas (4) y telesecundaria } \\
\text { (1) }\end{array}$ & $\begin{array}{l}\text { En la Bitácora, muestran niveles de apropiación más incipientes } \\
\text { que el resto de los grupos de escuelas, parecidos a los de las } \\
\text { escuelas adicionales. }\end{array}$ \\
\hline
\end{tabular}

Un dato importante es que el PAM ha llegado a escuelas por vías distintas a las del taller de inducción. Varios supervisores han emprendido el acompañamiento a las escuelas que lo han solicitado. Los resultados de dicha implementación no han sido óptimos hasta ahora, pero dan cuenta de la movilización social que genera en la región el modelo por sí mismo.

Cabe destacar que las visitas de observación realizadas a escuelas de organización completa han permitido distinguir que el PAM es un modelo que, con sus respectivas adecuaciones, funciona en diferentes contextos escolares. Y que, al igual que en las escuelas multigrado, los instrumentos referentes a la Participación democrática son los que más se han instalado. 


\section{LOGRO ACADÉMICO: PAM-PLANEA}

Aunque es el primer año de aplicación de la prueba PLANEA (y, por tanto, no se pueden establecer comparaciones de las escuelas PAM en el tiempo), los resultados sugieren que el trabajo sostenido con este modelo representa ventajas añadidas en cuanto al logro académico de las y los estudiantes.

El análisis de la información arroja que tanto en lenguaje y comunicación como en matemáticas, los resultados de las escuelas PAM son más favorables que en escuelas de la misma condición socioeconómica sin el programa. En matemáticas hay alrededor de 5\% más de estudiantes en el nivel IV, y 7\% menos en el nivel I.

GRÁFICA 1. Porcentaje de alumnado por nivel en PLANEA de escuelas PAM y otras del estado con la misma condición socioeconómica (primarias generales e indígenas) en lenguaje y comunicación, ciclo 2014-15

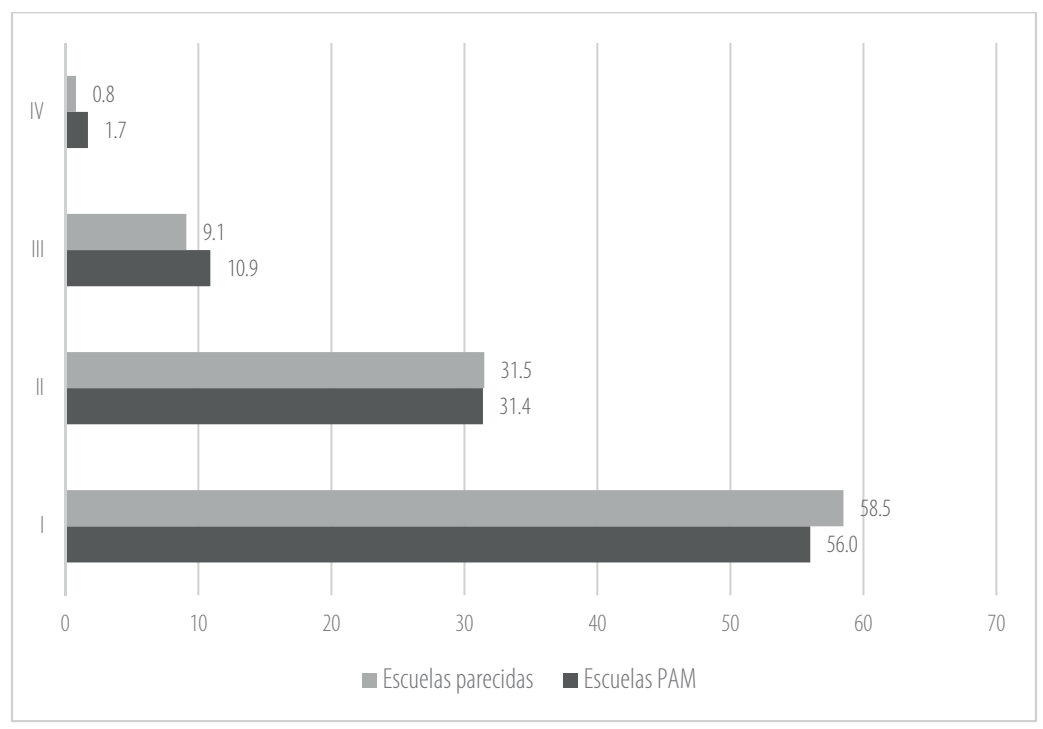


PROGRAMA DE APRENDIZAJE EN MULTIGRADO. UNA EXPERIENCIA DE MEJORA EDUCATIVA...

GRÁFICA 2. Porcentaje de alumnado por nivel en PLANEA de escuelas PAM y otras del estado con la misma condición socioeconómica

(primarias generales e indígenas) en matemáticas, ciclo 2014-15

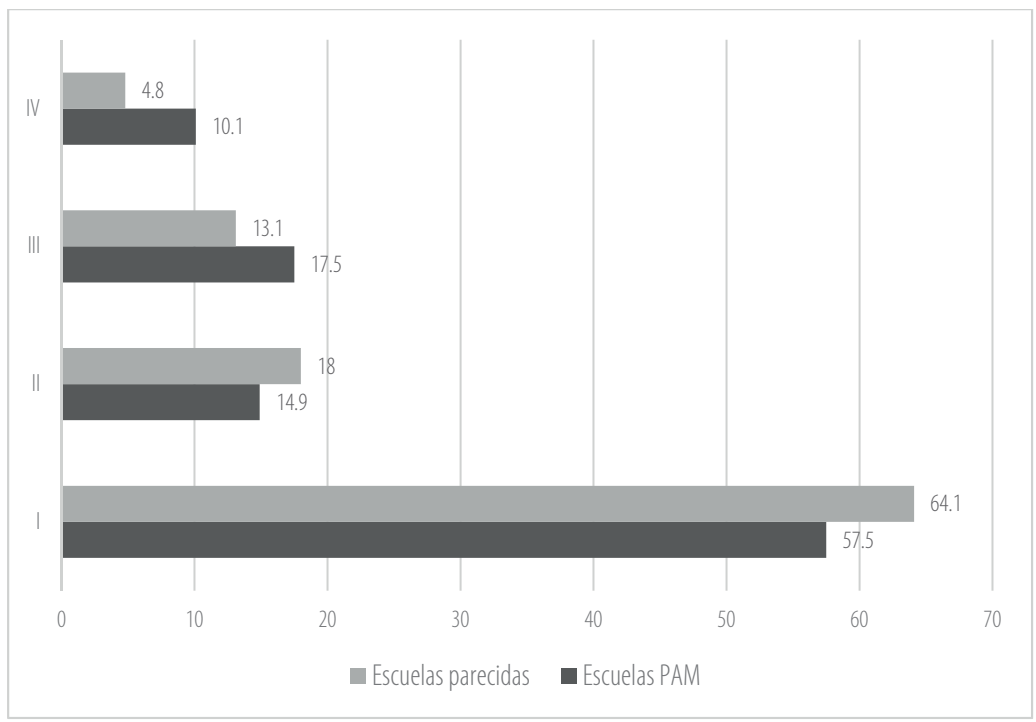

Al comparar las 15 primarias con más alto índice de implementación del PAM con las 15 de más bajo índice, también se observan diferencias importantes. En lenguaje y comunicación, hay casi $14 \%$ de estudiantes menos en el nivel I en las escuelas de alto índice. En matemáticas, los resultados son más favorables en las escuelas con alto índice en cada uno de los cuatro niveles. 
GRÁFICA 3. Porcentaje de alumnado por nivel en PLANEA, según índice de implementación del PAM (primarias generales e indígenas) en lenguaje y comunicación, ciclo 2014-15

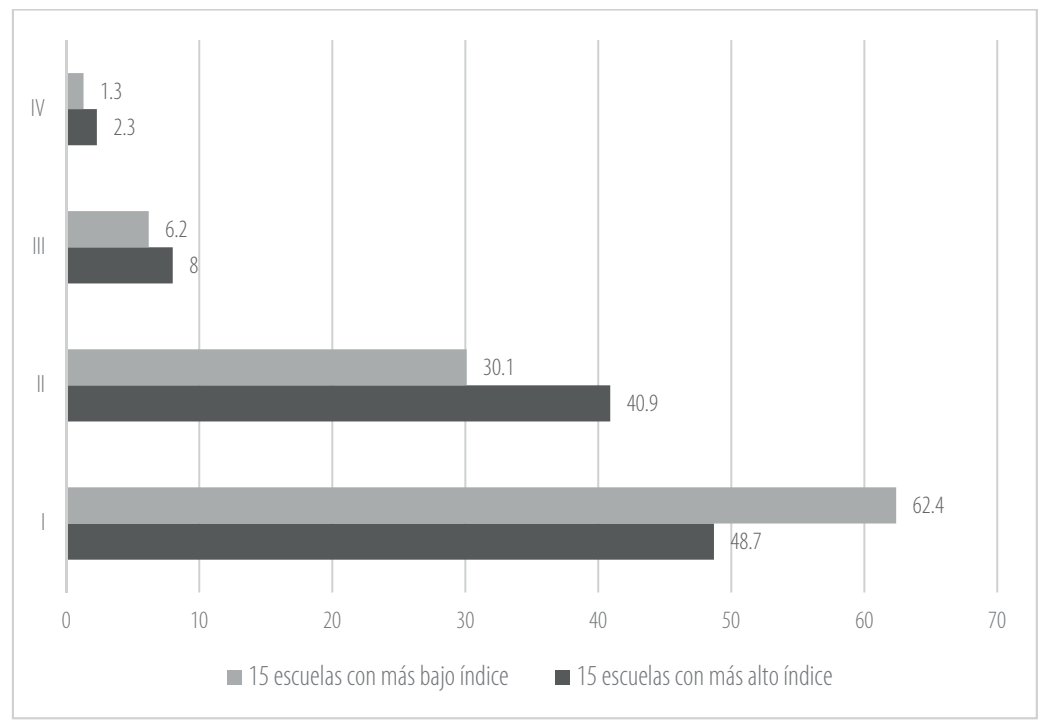

GRÁFICA 4. Porcentaje de alumnado por nivel en PLANEA, según índice de implementación del PAM (primarias generales e indígenas) en matemáticas, ciclo 2014-15

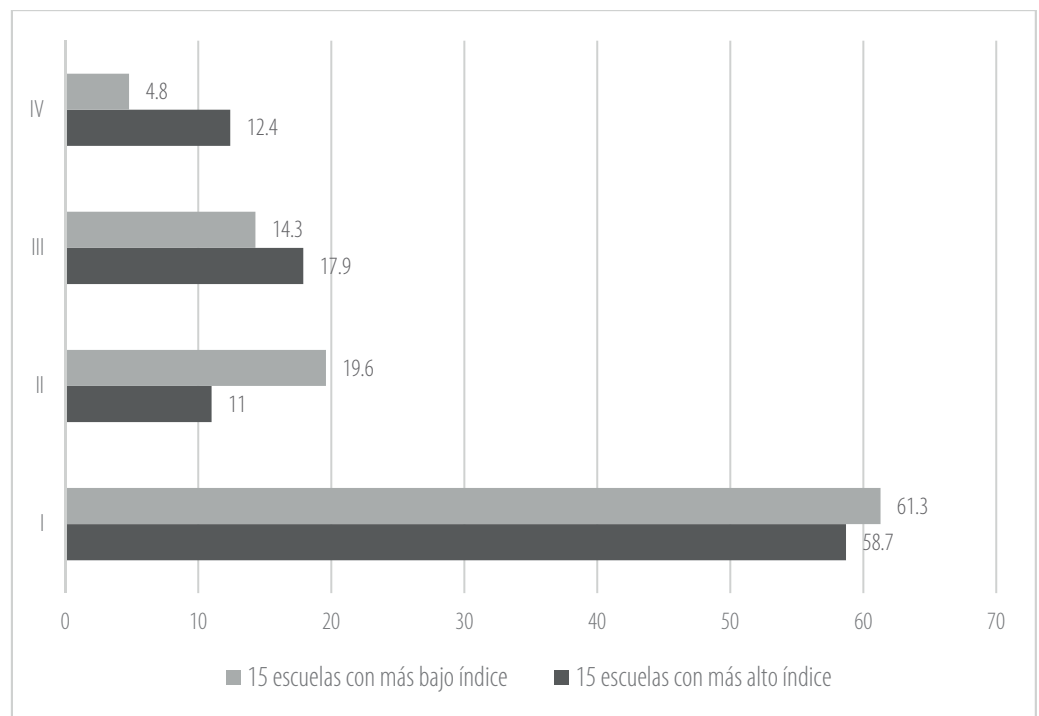


Finalmente, las primarias que han implementado el modelo durante tres ciclos (desde 2012-2013), muestran mejores resultados que las que lo han implementado durante un ciclo (2014-2015); sobre todo en lenguaje y comunicación, donde hay $11 \%$ menos estudiantes en el nivel I.

En resumen, las escuelas PAM tienen mejor desempeño académico que otras del estado de Puebla con la misma condición socioeconómica pues, entre más tiempo haya estado en el programa y este haya sido mejor implementado, mejores resultados tiene el alumnado en la prueba PLANEA.

\section{PERSPECTIVA DE FUTURO}

El PAM es un modelo que fortalece la escuela, no sustituye a otros, y dinamiza la propuesta pedagógica de la Secretaría de Educación Pública. Como se ha mostrado a lo largo del texto, el programa ha beneficiado de manera favorable a las escuelas que lo integraron en su quehacer. Es una propuesta específica de atención a las escuelas de organización multigrado, que fortalece su organización y la gestión del aprendizaje por parte del docente, pero que también da indicios de que ha incidido en el aprendizaje del alumnado.

La escuela multigrado es un modelo educativo en sí mismo, y hay que atenderlo como tal; no se trata de "escuelas incompletas". Igualmente $y$, dado el alto porcentaje de las tipo multigrado en el país, es menester brindarles una opción pertinente a sus condiciones y que facilite la mejora de la calidad educativa.

Es conveniente definir una estrategia de expansión gradual de este Programa a otras entidades, quizá focalizando a aquellas en las que haya un alto porcentaje de escuelas multigrado. Para ello, es imprescindible recuperar los aprendizajes y los resultados de las etapas de su implementación en el estado de Puebla.

Cabe destacar que el programa apuntala algunas consideraciones del Nuevo Modelo Educativo, presentado por la Secretaría de Educación Pública, en especial en los principios pedagógicos, explícitos en la Propuesta curricular para la educación obligatoria 2016, que son: 
- La autonomía curricular.

- La educación socioemocional.

- Reconocer la naturaleza social del conocimiento.

- Mejora de los ambientes de aprendizaje.

- Favorecer la cultura de aprendizaje.

- Apoyar en la transformación de la cultura pedagógica de la función docente.

- Ahondar el vínculo escuela/comunidad/familias.

El PAM, en efecto, hace énfasis en la importancia del aprendizaje colaborativo, en la responsabilidad sobre el propio proceso de aprendizaje por parte del estudiante, en los espacios de educación informal donde la familia y la comunidad cobran enorme relevancia, así como en el rol del docente, que con el PAM, se reconfigura tras el trabajo con los diversos instrumentos. Asimismo, empata con la promoción de Ambientes de aprendizaje como los factores que favorecen u obstaculizan la interacción social en los espacios educativos.

\section{REFERENCIAS BIBLIOGRÁFICAS}

Centro de Estudios Educativos (CEE). Informe Pretest. Monitoreo y Evaluación de la implementación del modelo Escuela Nueva en el estado de Puebla, México, CEE, 2013.

Centro de Estudios Educativos (CEE). Informe Postest. Monitoreo y Evaluación de la implementación del modelo Escuela Nueva en el estado de Puebla, México, CEE, 2013.

Centro de Estudios Educativos (CEE). Evaluación del Programa de Aprendizaje en Multigrado en el estado de Puebla. Informe Pretest, México, CEE, 2015.

Centro de Estudios Educativos (CEE). Evaluación del Programa de Aprendizaje en Multigrado en el estado de Puebla. Informe Pretest, México, CEE, 2015.

Estrada Rebull, María del Mar. "Multigrado en derecho propio", en Revista Latinoamericana de Estudios Educativos, XLV(2), abril-junio, México, CEE, 2015, pp. 43-62,. 
PROGRAMA DE APRENDIZAJE EN MULTIGRADO. UNA EXPERIENCIA DE MEJORA EDUCATIVA...

Instituto Nacional para la Evaluación de la Educación (INEE). Panorama educativo 2014. Indicadores del Sistema Educativo Nacional. Educación básica y media superior, México, INEE, 2015.

“Anexos electrónicos: PG01a-1. Porcentaje de escuelas preescolares unitarias que imparten los tres grados por tipo de servicios (2013-2014)".

"Anexos electrónicos: PG01b-1. Porcentaje de escuelas primarias de organización multigrado según entidad federativa y tipo de servicio (2013-2014)".

"Anexos electrónicos: PG01c-1. Porcentaje de escuelas telesecundarias unitarias y bidocentes que imparten los tres grados por entidad federativa (2013-2014)".

Instituto Nacional para la Evaluación de la Educación (INEE). Panorama Educativo de la Población Indígena, México, INEE, 2016.

Little, Angela. Education for All and Multigrade Teaching, Institute of Education, University of London, U. K., 2006.

McEwan, Patrick. La efectividad de las Escuelas Multigrado en Colombia, Stanford University, 1998.

Schiefelbein, E. En busca de la escuela del siglo XXI. ¿Puede darnos la pista la Escuela Nueva de Colombia?, Santiago de Chile, OREALC, 1993.

Secretaría de Educación Pública del estado de Puebla et al. Guía para la gestión escolar y del aula del Programa de Aprendizaje Multigrado en el estado de Puebla. Orientaciones para docentes, México, SEP Puebla, Fundación Azteca, Escuela Nueva México, CEE, 2014.

Secretaría de Educación Pública. Propuesta curricular para la educación obligatoria, 2016, México, SEP, 2016. 
